Paul Forchheimer

The category of person in language 



\section{THE CATEGORY OF \\ PERSON IN LANGUAGE}

by

\section{PAUL FORCHHEIMER}

Walter de Gruyter\& Co. vormals G. J. Göschen'sche Verlagshandlung - J. Guttentag, Verlaggbuchbandlung - Georg Reimer - Karl J. Trübner - Veit \& Comp.

Berlin 1953 
Arcbiv-Nr. 475453

Printed in Germany

Alle Rechte des Nachdrucks, der photomechanischen Wiedergabe, der Ubersotsang, der Heretellung von Pbotokopien und Mikrofilmen, auch ausugeweiee, vorbehalten.

Satz und Druck : Buchknnst, Berlin $\mathbf{W} 35$ 
IN MEMORIAM

PATRIS PRAECEPTORISQVE

PHILOSOPHIAE DOCTORIS IACOBI FORCHHEIMER

NATI IN MONTE NOBILITATIS FRANCONIAE INFERIORIS

DIE SEXTO AB CALENDIS IVNII MDCCCLXXVI ET MORTVI IN MONTE REGIO CANADENSE CALENDIS IANVARII MCMXLIV PRIMVM SVORVM LIBRORVM DEDICAVIT GRATVS FILIVS

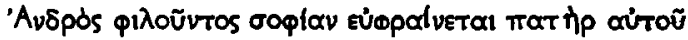

Prov. XXIX, 3 
